Research Article

\title{
Lightweight and High-Strength Design of an Excavator Bucket under Uncertain Loading
}

\author{
Xiujuan Yu $\mathbb{D},{ }^{1}$ Xiaoping Pang $\mathbb{D}^{1},{ }^{1}$ Zhihong Zou $\mathbb{D}^{1},{ }^{1}$ Guo Zhang ${ }^{D},{ }^{1}$ Ying $\mathrm{Hu}^{2}$ \\ Jingjun Dong $\mathbb{D}^{3}{ }^{3}$ and Houli Song $\mathbb{D}^{3}$ \\ ${ }^{1}$ College of Mechanical Engineering, Chongqing University, Chongqing 400044, China \\ ${ }^{2}$ School of Civil Engineering, Chongqing University, Chongqing 400044, China \\ ${ }^{3}$ Chongtong Chengfei Jiangsu Wind Energy Equipment Ltd., Jiangsu 518054, China
}

Correspondence should be addressed to Xiaoping Pang; pangxp@cqu.edu.cn

Received 8 July 2019; Revised 24 August 2019; Accepted 6 September 2019; Published 7 October 2019

Academic Editor: Sylwester Samborski

Copyright (c) 2019 Xiujuan Yu et al. This is an open access article distributed under the Creative Commons Attribution License, which permits unrestricted use, distribution, and reproduction in any medium, provided the original work is properly cited.

This paper presents a methodology for lightweight and high-strength design of an excavator bucket under uncertain loading. Uncertain loads are obtained by using the Monte Carlo simulation based on the existing soil-bucket interaction model in which the soil parameters are variable. And the well-known 3-sigma methodology is used for the quantification of the uncertain loads. Excavator bucket modelling is finished by using ANSYS Parameter Design Language (APDL). A multiobjective optimization model aiming to decrease the maximum von Mises stress and to reduce the weight of the bucket is established on the foundations of the uncertain load and the parametric geometry model. The structural shape and topology of the bucket are then designed by using the mixed variable genetic algorithm to solve the established optimization problem. The results show that the presented method can be effectively and efficiently applied for the optimization design of the excavator bucket and that the optimized bucket signifies obvious decreases in the weight and the stress compared with the initial reference model. The proposed methodology for structure optimization design considering uncertain loads not only provides the technical means for the design and development of high-performance bucket but also lays a preliminary theoretical foundation for the optimization design integrated machineenvironment interaction.

\section{Introduction}

Structure optimization design based on the deterministic knowledge and ignoring the machine-environment interaction is unable to satisfy the requirements on the high service performance and complex working condition of modern engineering structures. The digging operation using an excavator bucket is a typical and complex soil-bucket interaction process, in which the loads acting on the bucket are uncertain and the stresses in the bucket material are variable due to a constantly changing environment. The traditional deterministic design method takes no account of these uncertainties in loading condition and calculates the design loads under limiting working condition according to the theoretical or empirical load model [1-4]. Thus, the highstrength structures are designed at the expense of increased structure weight. However, from the perspectives of saving material and energy and prolonging service life, a lightweight and high-strength bucket structure is generally expected. Fortunately, the probability theory and statistical principle provide a sound mean to treat the uncertainties during the digging process. Furthermore, multiobjective optimization design with the consideration of uncertainties in loads has the potential to achieve the high-performance metrics of the bucket structure.

Based on the aforementioned background, the main aim of this research is to find an approach to treat the uncertainties in loads during the digging process and further to use this approach to guide the structure optimization design. Many soil-bucket interaction models have been established [5-8]. All these models provide the corresponding lumpedparameter functions to calculate the resistive force. More importantly, these lumped-parameter models lay a solid foundation for online soil-bucket interaction identification 
[9], resistive force prediction based on learning method [10], digging process simulation [11], and development of virtual excavation simulator [12] as well as dynamic motion planning for autonomous excavation [13]. An accurate and reliable soilbucket interaction model used to calculate the design loads is the prerequisite for the effective and credible optimization design of a bucket. Unfortunately, it is difficult to realistically model the soil-bucket interaction due to the complexity of geometric shape of the bucket and the uncertainity of interaction mechanism. Uncertainties in the soil parameters included in the models still cannot be eliminated, even if the well-known existing models are used to estimate the resistive forces. Among all previous works, resistive force prediction based on online learning [10] can identify and modify the model, but it is very time consuming and unsuitable for structure optimization. The existing digging simulation framework $[11,12]$ can only be used to generate deterministic load profiles for structure analysis and design. Some works $[1,14]$ provide the methods to determine the maximum theoretical digging forces which the excavator can apply in particular directions of the bucket force space. To quantify the uncertainity in digging resistance, Chen et al. [15] gave the most probable direction interval of digging resistance based on the experimental results and proposed a set of performance measures to characterize the digging performance of an excavator in this most probable interval. It is worth noting that these methods are used to assess an excavator's digging capability from the perspective of the driving side of the excavator. Thus, the digging capability metric calculated by using these methods is a theoretical limiting force and can only be used as a limiting load for the ultimate strength design of excavator structure. That is to say, these traditional methods only support relatively conservative structure design and do not allow for uncertainties inherent in the load model parameters. So far, the related research integrating the uncertain resistive force model into the bucket structure optimization design framework has not been reported. On the aspect of structure design, Qiu et al. [2] conducted the optimal design of an excavator's working device including the boom, stick, and bucket based on multiple surrogate models. The work of Qiu et al. [2] still belongs to the field of ultimate strength design. Rusinski et al. [3] conducted the research on the investigation and modernization of buckets of surface mining machines and presented an approach to consider the test results in real mining conditions as well as the numerical analysis results of modern numerical tools that support the bucket structure design in the process of bucket structure optimization. The existing researches on the bucket structure design mainly focus on ultimate strength design based on the deterministic limiting loads. In order to achieve a satisfactory trade-off between the weight and strength of a bucket, it is necessary to conduct a profound study on the uncertainity optimization design of the bucket structure with the consideration of uncertainties in the soil-bucket interaction process.

This paper takes the soil-bucket interaction into consideration during the process of bucket optimization design. Uncertainties inherent in soil parameters are represented by using the Monte Carlo method to simulate the random distribution characteristics of parameters in uncertain intervals. The existing Park's soil-bucket model [7] is extended to be used in uncertain condition. Furthermore, to quantify the uncertainity of the resistive forces acting on the bucket and obtain the design load indicators with a relatively great possibility and low computational cost, the 3-sigma methodology which has been widely used in robust design optimization of engineering structure [16-18] is introduced to define the level of load dispersion and determine the design loads. An excavator bucket model with complex geometrical configuration is parametrically represented and modelled by using APDL so as to achieve design automation and integration with a variety of industrial software products. Finally, a framework for the lightweight and highstrength design of an excavator bucket with the integration of uncertain soil-bucket interaction model is developed. The implementation of this optimization framework into the established multiobjective optimization of an excavator bucket is also shown.

\section{Soil-Bucket Interaction Modelling and Uncertainity Quantification}

The digging process is affected by many variable factors, such as rough and changing terrains, uncertain soil properties, complex geometric shape of the bucket, and different operation styles used by operators who have different operating preferences and experiences. Therefore, it is difficult to establish a comprehensive and accurate theoretical model of the excavator digging process that can realistically reflect the soil-bucket interaction mechanism. To obtain the information of variability in operation, uncertainity in soil properties, and fluctuations in environment, and further to achieve human-machine-environment fusion, some external detection apparatus (transducers, radar detectors, and cameras) have been widely used in autonomous excavation [19-21]. However, during the design phase, all these uncertain information cannot be obtained; thus, a model that can be used to realistically simulate the digging process is essential for determination of design loads of bucket design. Traditional theoretical digging forces are defined as the forces generated at the bucket lip or cutting edge when operating the bucket or stick cylinders independently [1], as shown in Figure 1. The traditional bucket digging force $F_{b d}$ and stick digging force $F_{S d}$ are calculated by taking into consideration hydraulic pressure limit and excavator stability limits including tipping and slipping limits. Since the soil-bucket interaction relationship is oversimplified in the traditional method, it cannot be used to guide the detailed design of the bucket structure. Among all developed soilbucket interaction models, Park's resistance model [7] is selected in this research as the basic model to predict the soil resistive forces. Park's model [7] is better suited to simulate the soil-bucket interaction for the following reasons:

(1) It is a 3D analytical resistance model extended based on Perumpral's model [22], whose soil failure model is close to 3D soil failure geometries.

(2) It incorporates the excavator bucket shape. The influences of a bucket's constitutive parts such as two side 


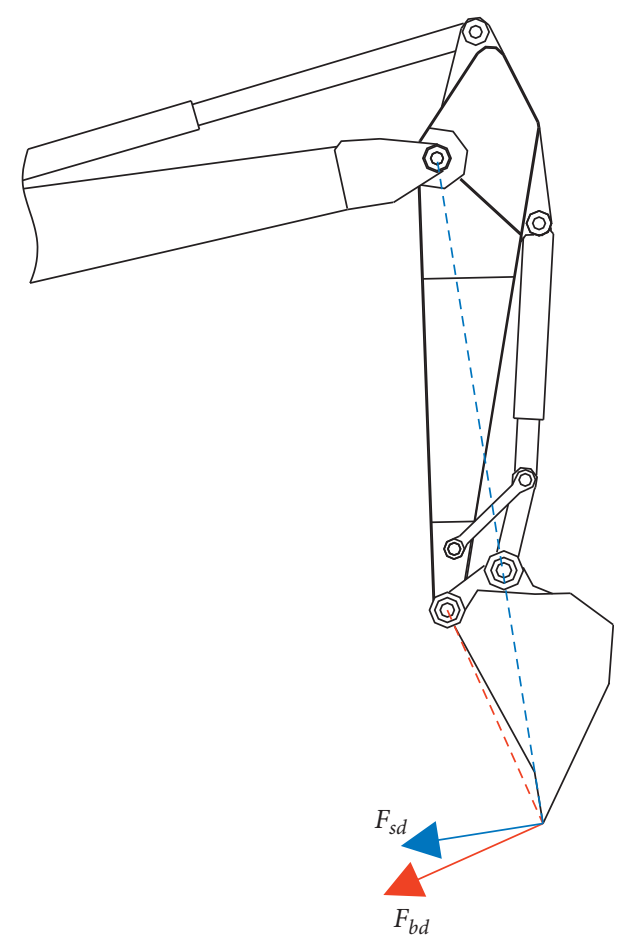

FIgURE 1: Theoretical digging forces.

plates and cutting plate on the soil-bucket interaction can be analysed and modelled individually and then added to the resultant resistance force by solving the limit equilibrium equations of the $3 \mathrm{D}$ soil failure wedge. Resistance model adding the influences of bucket shape can be used to determine the design loads in the process of bucket structure design.
2.1. Soil-Bucket Interaction Model. In Park's resistance model [4], the soil rupture surface is assumed as a flat shape and the separated soil body is considered as an idealized failure wedge, as shown in Figure 2. Figure 2(b) illustrates the soil failure wedge and all the forces acting on its surfaces. Among all constitutive surfaces, the surfaces $\square$ acfd and $\square b c f e$, respectively, represent the terrain surface and the failure surface, the surface $\square$ abed is the contact surface between the bucket cutting plate and soil failure wedge, and the surfaces $\triangle a b c$ and $\triangle d e f$ are the side rupture surfaces. Figure 2(a) illustrates a bucket sketch, in which the surfaces $\triangle a b g$ and $\triangle d e h$ are both the contact surfaces between the soil failure wedge and the side plates of the bucket. In this resistance model, the force components acting on the wedge are completely considered. These force components include the cohesion force $\mathrm{CF}_{1}$ acting on the failure surface $\square b c f e$, the frictional forces $\mathrm{SF}_{1}$ and $\mathrm{SF}_{2}$, respectively, acting on the surface $\square b c f e$ and the side rupture surfaces $\triangle a b c$ and $\triangle d e f$, the adhesion force $F_{a d}$ acting on the contact surface $\square$ abed, the adhesion-cohesion force ACF acting on the two side rupture surfaces, and the gravitational force $W$ and the surcharge $Q$. All factors which have effects on the resistive force are considered except for the inertia force compared with the previous resistance model when digging speed is low or not considered. In addition, the forces $F_{N}$ and $F_{r 2}$ are the normal forces, respectively, acting on the failure surface $\square b c f e$ and the contact surface $\square a b e d$, and the force $R_{S}$ is the resistive force acting on the contact force $\square$ abed. The resistive force $R_{S}$ can be solved by conducting the static equilibrium analysis for the soil failure wedge, as follows:

$$
R_{S}=\frac{-F_{a d} \cdot \cos (\beta+\rho+\varnothing)+(W+Q) \cdot \sin (\alpha+\rho+\varnothing)+\left(2 \mathrm{SF}_{2}+2 \mathrm{ACF}+\mathrm{CF}_{1}\right) \cdot \cos \varnothing}{\sin (\beta+\rho+\delta+\varnothing)}
$$

where $\alpha$ is the slope angle of the terrain surface, $\beta$ is the angle between the cutting plate and the terrain surface, $\rho$ is the soil failure angle, and $\delta$ and $\varnothing$ are, respectively, the soil-metal friction angle and soil internal friction angle. In equation (1), all the individual force components have the same representations with the corresponding terms in Park's model [4] except for the term $Q$ representing the surcharge. For the sake of simplification, similar terms will not be listed in this research. The surcharge $Q$ is determined by the volume of the soil swept by the bucket, and its representation is written as

$$
Q=r \cdot g \cdot V_{S}
$$

where $r$ is the soil density, $g$ is the gravitational acceleration, and $V_{S}$ is the volume of the soil swept by the bucket.

According to the action and reaction relations of all force components, the forces acting on the bucket are also given in Figure 2(a). From Figure 2(a), it can be seen that the forces acting on the bucket cutting plate include the adhesion force
$F_{a d}$ and the resistive force $R_{S}$, and the side plates of the bucket encountered the adhesion force $F_{a d 1}$ and the normal force $F_{r}$. The expression of the resistive force $R_{S}$ has been given in equation (1), and the other force components acting on the bucket can be determined by the following formulas:

$$
\begin{aligned}
S_{\square a b e d} & =l_{a b} \cdot l_{b e}, \\
F_{a d} & =c_{a} \cdot S_{\square a b e d}, \\
S_{\Delta a b g} & =\frac{l_{a b}^{2} \cdot \sin \beta \cdot \sin \varepsilon}{2 \sin (\beta+\varepsilon)}, \\
F_{a d 1} & =c_{a} \cdot S_{\Delta a b g}, \\
F_{r} & =k_{0} \cdot r \cdot g \cdot z \cdot S_{\Delta a b g} .
\end{aligned}
$$




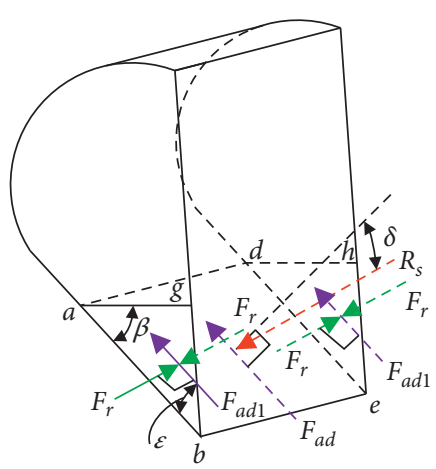

(a)

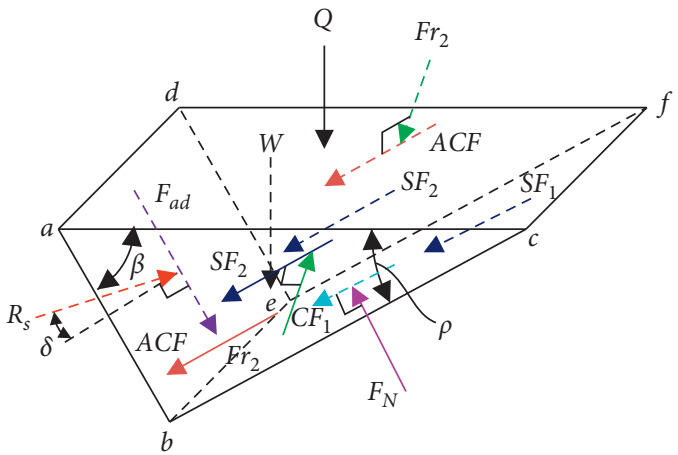

(b)

Figure 2: Soil-bucket interaction model. (a) Bucket sketch; (b) soil failure wedge and forces.

In above equations, $l_{a b}$ and $l_{b e}$, respectively, represent the length of bucket cutting plate in contact with the soil and the bucket width, $S_{\square a b e d}$ and $S_{\triangle a b g}$ are the areas, respectively, of the contact surfaces $\square$ abed and $\triangle a b g, c_{a}$ is the soil adhesion, $k_{0}$ is the coefficient of lateral earth pressure at rest, $z$ is the depth from the terrain surface to the centroid of the contact surface $\triangle a b g$, and $\varepsilon$ is the angle between the rim of the side plate and the cutting plate of the bucket, and $\varepsilon$ is also called the blunt edge angle.

So far, the soil-bucket interaction model has been established in detail based on Park's model [7]. This model includes the terms representing the interaction between the soil and the constitutive parts of the bucket, and gives the detailed expressions of the force components acting on the bucket. Thus, it contributes to the detailed design of the bucket with complex geometric shape.

\subsection{Uncertainity Quantification and 3-Sigma Methodology.}

There are three types of uncertainity in the digging process: the variability in soil properties, changeability in operating styles, and imperfection and inaccuracy in model. In this research, our focus is on the influences of the uncertain soil properties on the force components acting on the bucket to further determine the design loads for structure optimization. Although the operating styles of operators are reflected by the real-time manipulator configurations of an excavator, in the soil-bucket interaction model operating styles can be simply described by the digging angle $\beta$ and the digging depth $d$. According to Figure 2(b), the digging angle $\beta$ is the angle between the cutting plate and the terrain surface, and the digging depth is the distance from the cutting edge be to the terrain surface $\square$ acfd. The digging depth can be calculated by

$$
d=l_{a b} \cdot \sin \beta .
$$

To investigate the variation of each force component acting on the bucket for the operation parameters, the values of all these force components are calculated for different digging angles and digging depths. Considering the structure characteristic of the bucket, the maximum length of $l_{a b}$ is taken as 0.6 ; thus, the variation range of $l_{a b}$ is $[0,0.6]$. The variation interval of the digging angle $\beta$ is set as $\left[0,90^{\circ}\right]$. Due to the assumption of soil failure wedge, for any given digging angle the digging depth should be subjected to the following geometric constraint:

$$
d \leq l_{a b \max } \cdot \sin \beta,
$$

where $l_{a b \max }$ is the maximum value of the length of bucket cutting plate in contact with the soil, i.e., $l_{a b \max }=0.6$. Figure 3 shows the variation of the force components under different operation parameters, and the parameters used for the simulating calculation of the force components are outlined in Table 1. It must be noted that all these parameters used to calculate the forces are deterministic. For the soil failure angle $\rho$, Zou et al. [4] gave the following approximation expression:

$$
\rho=\left(45^{\circ}-\frac{\varnothing+\delta}{2}\right)+\left(45^{\circ}-\frac{\beta}{2}\right) .
$$

In Figures 3(a)-3(d), it can be noted that a larger digging depth generates larger forces if the digging angle is kept the same. However, the effects of the digging angle on the forces are not the same when the digging depth is fixed. In Figure 3(a), with any fixed digging depth, the smaller digging angles correspond to the larger adhesion force $F_{a d}$. In Figure 3(d), the resistive force $R_{S}$ increases with the increase in the digging angle, but the adhesion force $F_{a d 1}$ and the normal force $F_{r}$ have larger values when the digging angle is close to its interval boundaries shown in Figures 3(b) and $3(c)$. In general, the maximum values of all these forces are obtained when the digging angle and digging depth are, respectively, close to their maximum values. This means that the effect of the digging depth on all these forces is dominant compared with the effect of the digging angle. Among all the force components acting on the bucket, the resistive force $R_{S}$ is several orders of magnitude larger than the other forces. Therefore, the resistive force $R_{S}$ has the major contribution to the bucket damage during the digging process.

It is worth emphasizing that the above discussion about the relations between the forces acting on the bucket and the operation parameters is based on the deterministic soil characteristics. Unfortunately, uncertainties inherent in the soil properties cannot be ignored in the realistic 


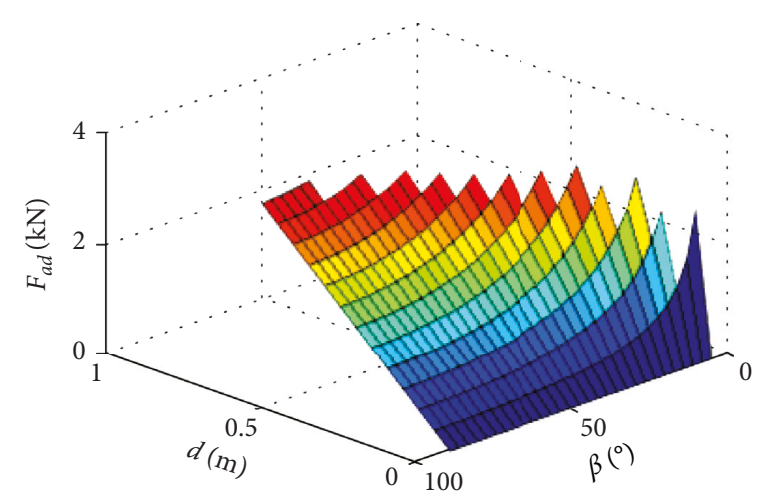

(a)

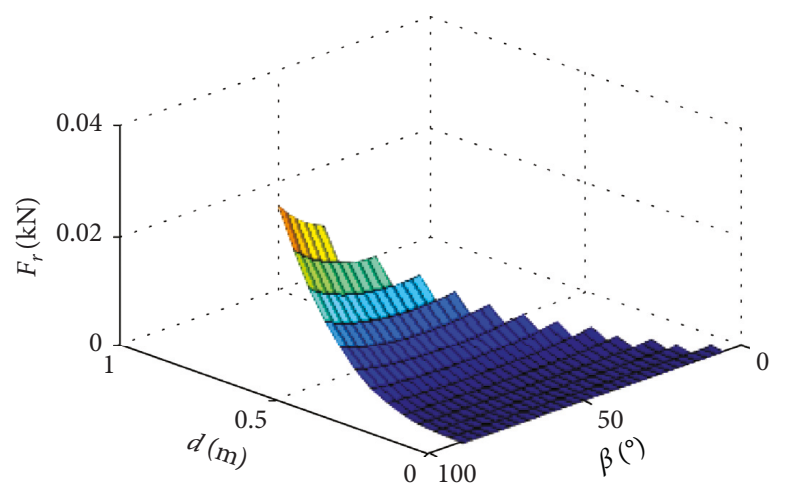

(c)

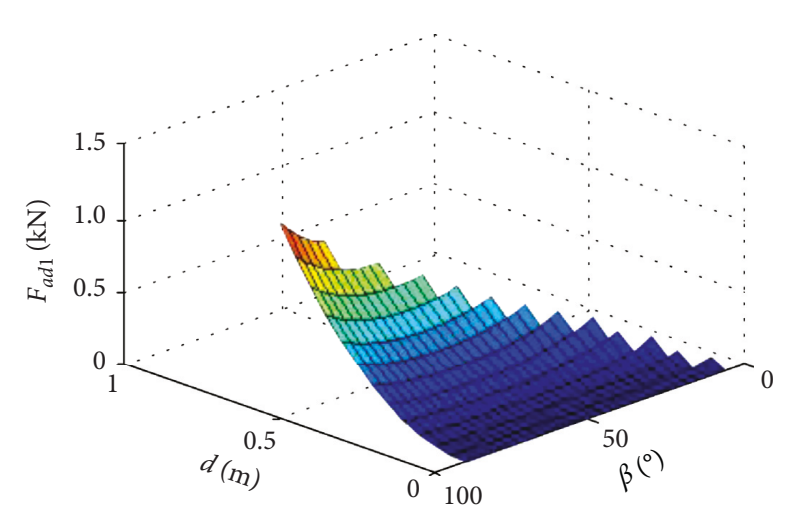

(b)

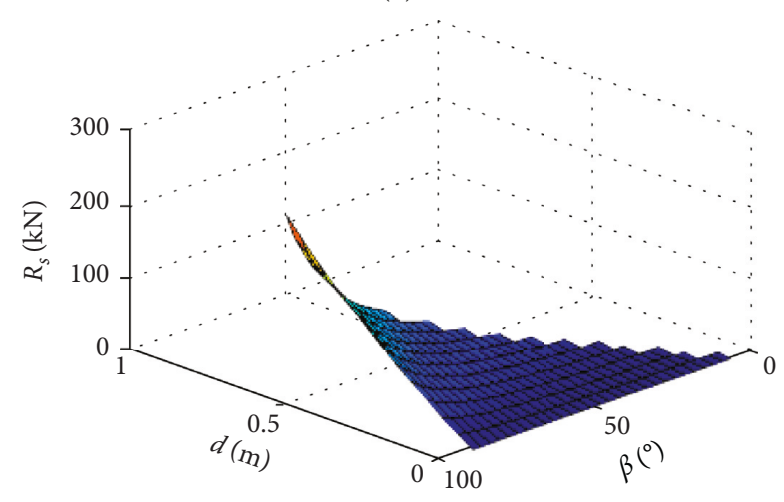

(d)

FIGURE 3: Force variation for digging depth and digging angle. (a) $F_{a d}$ with different $d$ and $\beta$, (b) $F_{a d 1}$ with different $d$ and $\beta$, (c) $F_{r}$ with different $d$ and $\beta$, and (d) $R_{S}$ with different $d$ and $\beta$.

TABle 1: Parameters used for the calculation of the force components [11].

\begin{tabular}{lccc}
\hline Parameter & Symbol & Value & Unit \\
\hline Soil density & $r$ & 2050 & $\mathrm{~kg} / \mathrm{m}^{3}$ \\
Soil internal friction angle & $\varnothing$ & 35 & $\circ$ \\
Soil adhesion & $c_{a}$ & 5000 & $\mathrm{~Pa}$ \\
Soil terrain inclination angle & $\alpha$ & 0 & $\circ$ \\
Soil cohesion & $c$ & 22000 & $\mathrm{~Pa}$ \\
Soil-bucket friction angle & $\delta$ & 23.3 & $\circ$ \\
Coefficient of earth pressure at rest & $k_{0}$ & 0.55 & - \\
Bucket width & $l_{b e}$ & 1.11 & $\mathrm{~m}$ \\
Blunt edge angle & $\varepsilon$ & 53 & $\circ$ \\
\hline
\end{tabular}

digging process. These uncertainties will lead to the variability of the forces. Especially for the forces which are sensitive to the variation of the soil properties, drastic force fluctuations will generate. Hence, the variability of the soil properties due to unstructured environment and heterogeneous soil medium is taken into account here. It is assumed that soil parameters are the members of the uncertain intervals. The upper and lower limits of uncertain intervals of the corresponding parameters are listed in Table 2.

For any fixed digging operation parameters, the calculation of the maximum values of all forces acting on the bucket is the process of solving the optimization problems under the constraints of the uncertain intervals of the soil parameters. The obtained forces by optimization are the global maximum values, and their occurrence probability is very low. If these maximum forces are used as the criteria for the bucket optimization, the design result will be conservative. In addition, if the calculation process of these forces is embedded into the bucket optimization process, the computation cost is unbearable. With these considerations in mind, in this research, 3-sigma approach [17-19] is introduced to quantify the dispersion level of the forces acting on the bucket under the influence of the uncertain soil properties. In the robust design optimization, the aim is to enable the range of $\pm n$-sigma around the mean value of the performance metric includes in an acceptable range [19]. Figure 4 shows the $\pm n$-sigma concept. It can be easily seen that the probability of belonging to the interval $[\mu-3 \sigma, \mu+3 \sigma]$ is 0.9973 , and the probability of belonging to the interval $[-\infty, \mu+3 \sigma]$ is 0.9987 . This probability is enough in engineering analysis and design. Thus, the process to quantify the loads uncertainty can be summarized as following two steps:

(1) Sample $N(N=10000)$. Samples of each of forces acting on the bucket. The Monte Carlo method is used to calculate the force components on the uncertain soil parameter intervals.

(2) Quantify the uncertain loads. The mean value $\mu$ and the standard deviation $\sigma$ of each force are calculated based on the results of the Monte Carlo simulation. 
TABLE 2: Uncertain parameters and corresponding limits.

\begin{tabular}{|c|c|c|c|c|}
\hline Parameter & Symbol & Lower limit & Upper limit & Unit \\
\hline Soil density & $r$ & 1650 & 2250 & $\mathrm{~kg} / \mathrm{m}^{3}$ \\
\hline Soil internal friction angle & $\varnothing$ & 25 & 35 & $\circ$ \\
\hline Soil adhesion & $c_{a}$ & 4500 & 5500 & $\mathrm{~Pa}$ \\
\hline Soil terrain inclination angle & $\alpha$ & 0 & 0 & $\circ$ \\
\hline Soil cohesion & $c$ & 18000 & 24000 & $\mathrm{~Pa}$ \\
\hline Soil-bucket friction angle & $\delta$ & 13.3 & 26.3 & $\circ$ \\
\hline Coefficient of earth pressure at rest & $k_{0}$ & 0.55 & 0.55 & - \\
\hline
\end{tabular}

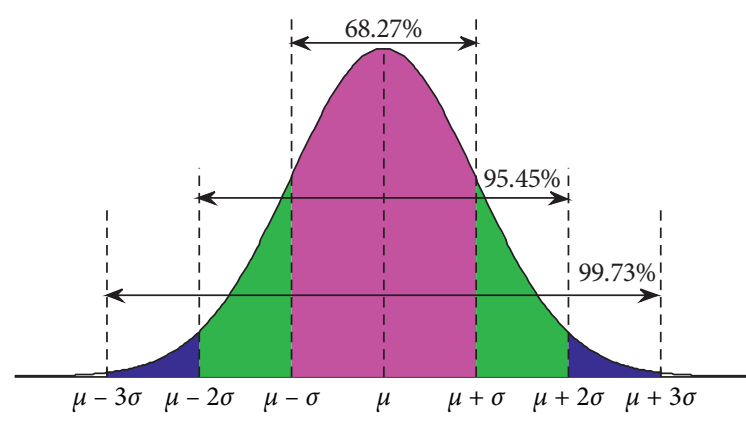

FIGURE 4: $\pm n$-sigma concept.

According to the $+3 \sigma$-sigma principle, the upper limits of all forces are taken as $\mu+3 \sigma$. This means that if these force limits are used as the design loads, the safety probability of the bucket structure is 0.9987 .

Figure 5 shows the Monte Carlo simulation results of all forces. The upper limits $\mu+3 \sigma$, mean value $\mu$, and the lower limits $\mu-3 \sigma$ of these forces have the same variation trends with the deterministic results. The maximum values of the upper limits of these forces are obtained when the digging depth and the digging angle are taken as their corresponding maximum values. Thus, the design loads of bucket optimization are determined by the upper limits $\mu+3 \sigma$ of all these forces with the maximum digging depth and digging angle. This means that the safety probability of the optimized bucket based on these design loads is 0.9987, and the computation cost is reduced considerably.

\section{Parametric Modelling and Static Strength Analysis of Excavator Bucket}

Figure 6 shows the schematic diagrams of the bucket. In Figure $6, t_{i}(i=1,2, \ldots, 5)$ represent the thickness parameters of the constitutive plates of the bucket, $l_{i}(i=$ $1,2, \ldots, 12)$ represent the length parameters, $\theta_{i}(i=1,2,3)$ are the angle parameters, and $r_{1}$ and $r_{2}$ are the radius parameters of the arc plates. Optimization process of the bucket involves design automation, integration of multiple industrial software products, and data exchange of different software products; thus, APDL is used for parametric geometry modelling of the bucket. APDL command flow has good portability and benefits structure reanalysis, so it is suitable for the optimization design of the complex bucket structure. Figure 7 gives the solid bucket model obtained by using APDL. Table 3 lists material properties of the bucket.

To investigate the relations of the structure stress and the loads based on the parametric bucket model, finite element analysis (FEA) under a series of deterministic load groups are conducted. As shown in Figure 6, joints A and B are the hinge points connecting the stick and bucket; thus, their axial degrees are fixed and the rest of degrees are released. It is assumed that all the force components acting on the bucket (as shown in Figure 2) are distributed uniformly on the corresponding contact surfaces between the soil and bucket. That is to say, all the forces are applied to the corresponding acting surfaces in the form of pressures. Figure 8 shows the variation of the maximum structure stress $S_{\max }$ for the forces acting on the bucket. There are the analysis results of 8 groups of deterministic load cases. In Figure 8, LG1 LG8 are the labels of the load cases. It is obtained that the larger resistive loads will result in the larger maximum structure stress. It can also be summarized that the resistive force $R_{S}$ is the major component influencing the magnitude of the maximum structure stress. The load group LG8 with the maximum digging depth and digging angle is listed in Table 4, and the FEA results under this load condition are given in Figure 9. The maximum stress of the bucket structure exceeds the yielding stress according to the analysis results; thus, plastic deformation will occur on the bucket. Improved design of the bucket can enhance the structure strength.

The established geometry model using APDL in this section provides a solid foundation for the optimization design of an excavator bucket. In addition, it is validated that the maximum structure stress and the loads acting on the bucket are positive correction. This further verifies that it is rational to use the upper limits $\mu+3 \sigma$ of the loads acting on the bucket with the maximum digging depth and digging angle as the design loads.

\section{Optimization Design of an Excavator Bucket}

To achieve lightweight and high-strength optimization of an excavator bucket under uncertain loading, a framework combining the mathematical software MATLAB and finite element analysis software ANSYS is established as shown in Figure 10. The MATLAB part of the framework consists of a soil-bucket interaction model module, Monte Carlo simulation module, uncertain loads quantification and processing module, and genetic algorithm (GA) module. This part is mainly used for determination of design loads 


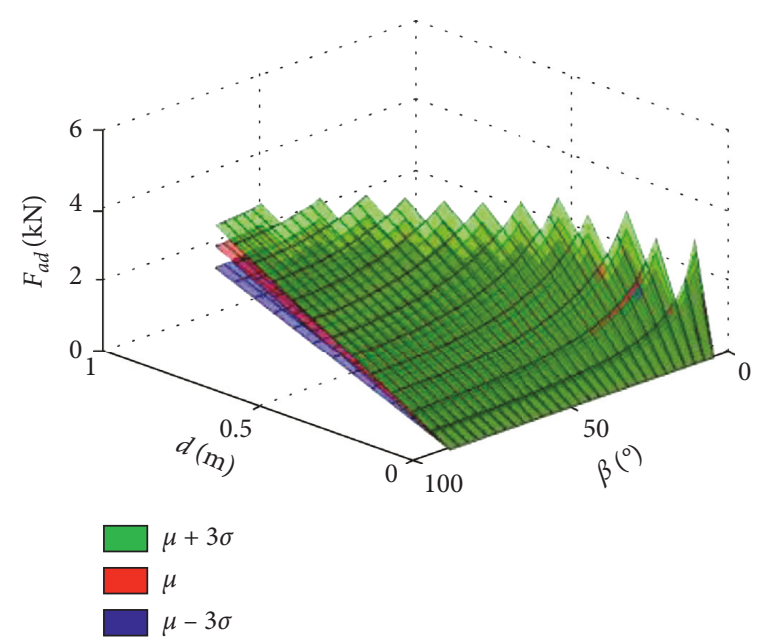

(a)

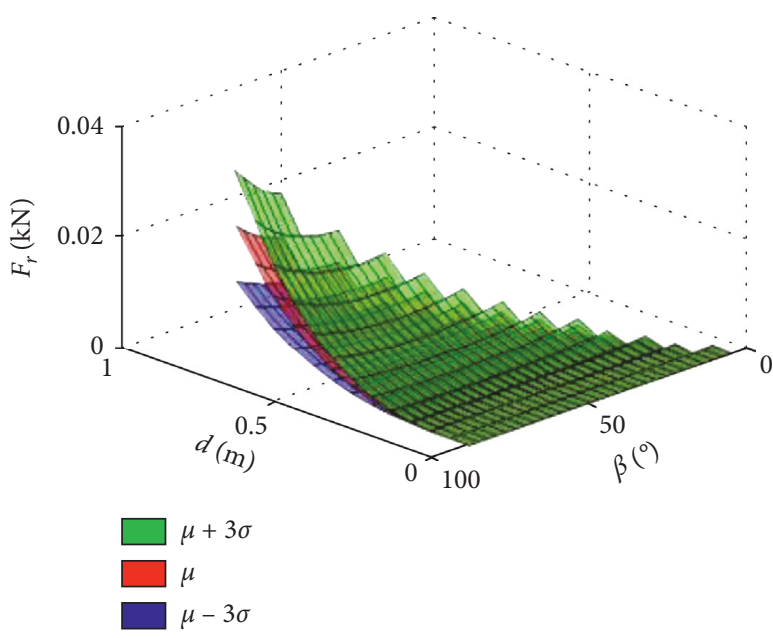

(c)

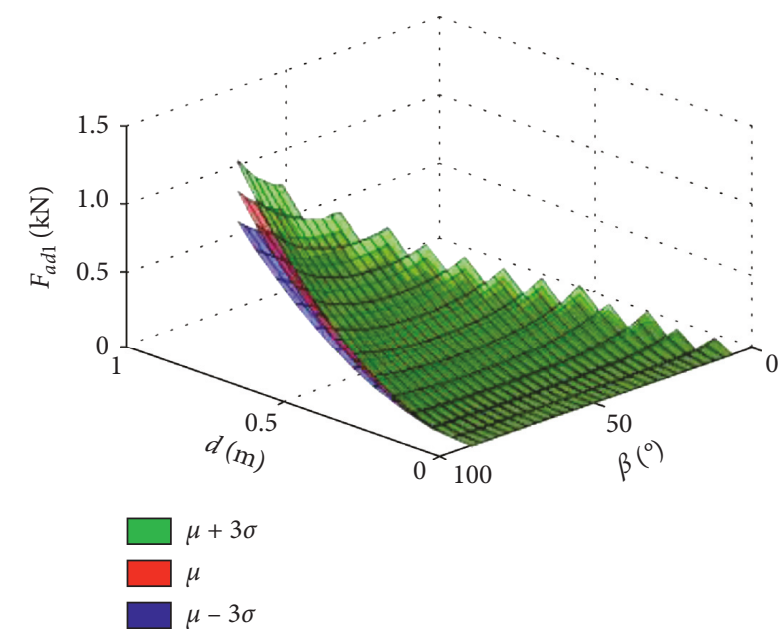

(b)

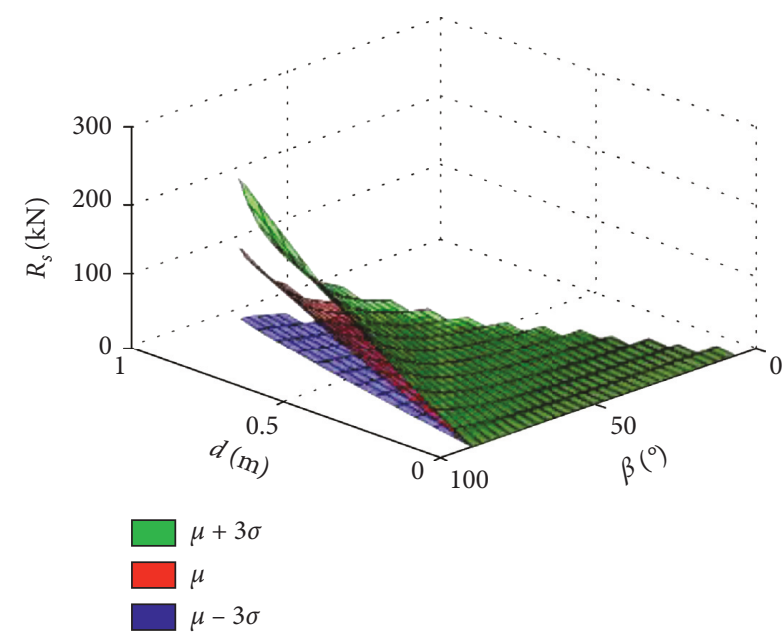

(d)

Figure 5: Monte Carlo simulation results of the forces. (a) $F_{a d}$ with different $d$ and $\beta$ under $\pm n$-sigma, (b) $F_{a d 1}$ with different $d$ and $\beta$ under $\pm n$-sigma, (c) $F_{r}$ with different $d$ and $\beta$ under $\pm n$-sigma, and (d) $R_{S}$ with different $d$ and $\beta$ under $\pm n$-sigma.

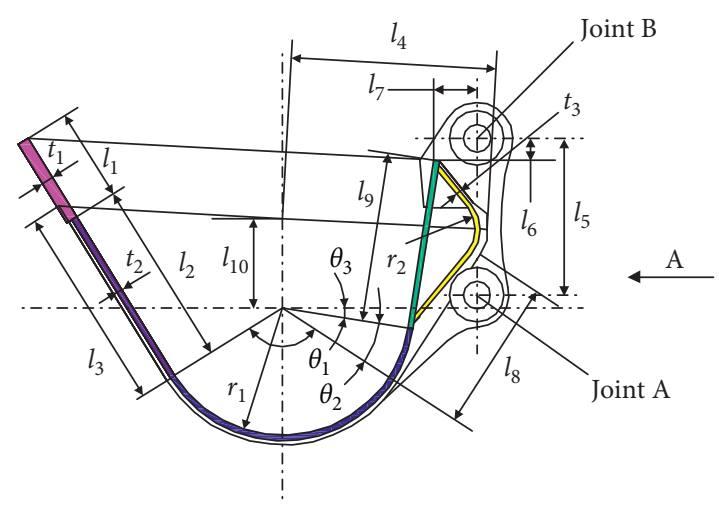

(a)

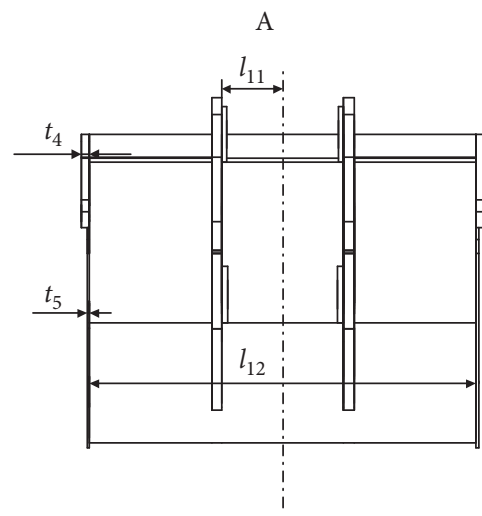

(b)

FIGURE 6: Schematic diagrams of the bucket. 


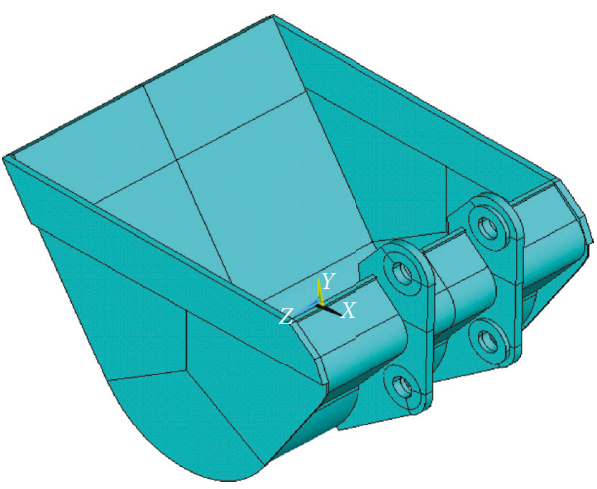

FIgURE 7: Solid bucket model using APDL.

TABLE 3: Material properties of the bucket [2].

\begin{tabular}{lcccc}
\hline Material & $\begin{array}{c}\text { Density } \\
\left(\mathrm{kg} / \mathrm{m}^{3}\right)\end{array}$ & $\begin{array}{c}\text { Poisson's } \\
\text { ratio }\end{array}$ & $\begin{array}{c}\text { Young's } \\
\text { modulus }(\mathrm{MPa})\end{array}$ & $\begin{array}{c}\text { Yielding } \\
\text { stress }(\mathrm{MPa})\end{array}$ \\
\hline ST345 & $7.85 \times 10^{3}$ & 0.3 & $2.06 \times 10^{5}$ & 345 \\
\hline
\end{tabular}

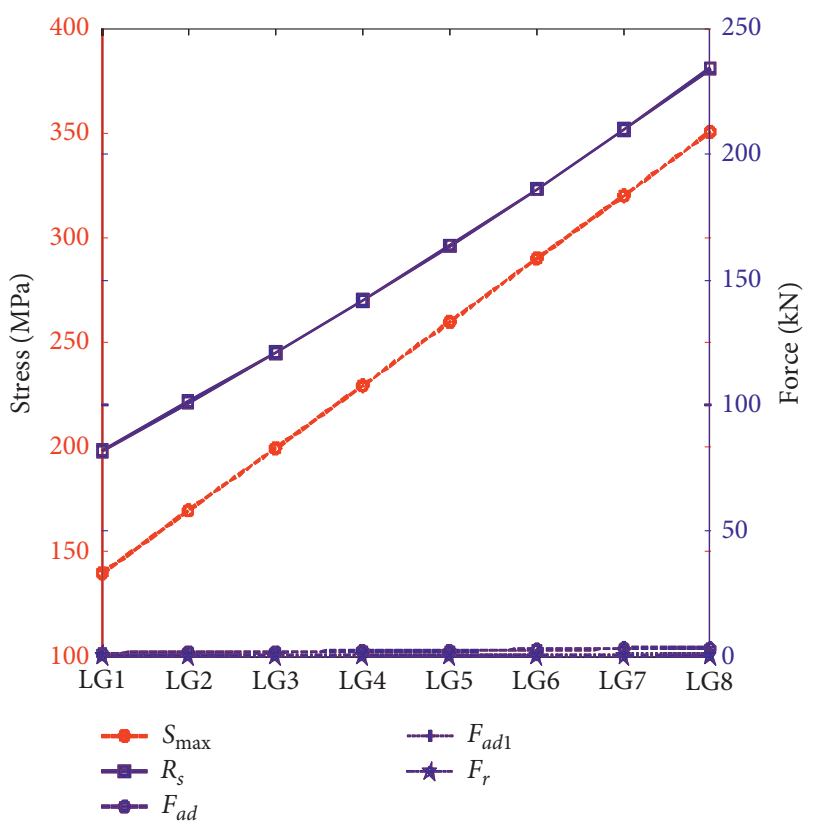

Figure 8: The relations of structure stress and loads.

based on the uncertain soil-bucket model and solving of the multiobjective optimization problem. The ANSYS part of the framework includes the parametric modelling module and FEA module. The remodelling module is called to remodel the new geometry structure during optimization process. Output and input modules are also included in the framework to read in and write out parameters.

Reduction of the maximum structure stress can decrease the structure damage and extend the structure service life. Besides, a lightweight structure can decrease energy cost and increase fuel economy of an excavator. Thus, the lightweight
TABLE 4: Deterministic loads with the maximum digging depth and digging angle (LG8).

\begin{tabular}{lcccc}
\hline $\begin{array}{l}\text { Load } \\
\text { name }\end{array}$ & $\begin{array}{c}\text { Resistive } \\
\text { force } R_{S} \\
(\mathrm{kN})\end{array}$ & $\begin{array}{c}\text { Adhesion } \\
\text { force } F_{a d 1} \\
(\mathrm{kN})\end{array}$ & $\begin{array}{c}\text { Adhesion } \\
\text { force } F_{a d} \\
(\mathrm{kN})\end{array}$ & $\begin{array}{c}\text { Normal } \\
\text { force } F_{r} \\
(\mathrm{kN})\end{array}$ \\
\hline Value & 233.950 & 1.194 & 3.330 & 0.032 \\
\hline
\end{tabular}

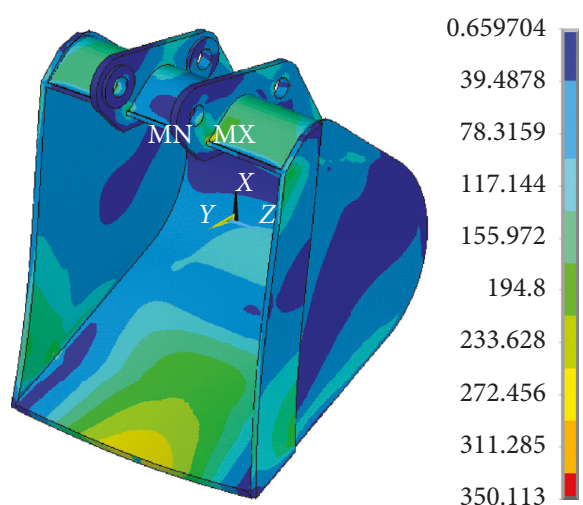

(a)

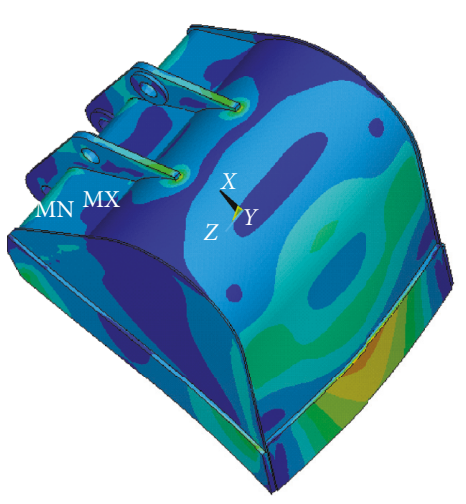

0.659704

39.4878

78.3159

117.144

155.972

194.8

233.628

272.456

311.285

350.113

(b)

Figure 9: FEA results based on the deterministic load group LG8.

and high-strength optimization model for the bucket under the action of the design loads determined by $3 \sigma$ methodology can be obtained as

$$
\begin{gathered}
\min _{\mathbf{X}} \frac{S_{\max }}{S_{\text {max,ref }}}+\frac{m}{m_{\text {ref }}}, \\
\text { s.t. } \quad \mathbf{X}_{L} \leq \mathbf{X} \leq \mathbf{X}_{U}, \\
\\
\quad V(\mathbf{X}) \geq V_{0},
\end{gathered}
$$

where $S_{\max }$ is the maximum structure stress due to the action of the upper limits $\mu+3 \sigma$ of the loads with the maximum digging depth and digging angle, $S_{\text {max,ref }}$ is the reference value of the maximum stress, $m$ is the mass of the bucket, $m_{\text {ref }}$ is the reference value of the bucket mass, $\mathbf{X}$ is the set of design variables which are given in Figure 6, and $\mathbf{X}_{L}$ and $\mathbf{X}_{U}$ are, respectively, the lower and upper bounds of $\mathbf{X}, V(\mathbf{X})$ is 


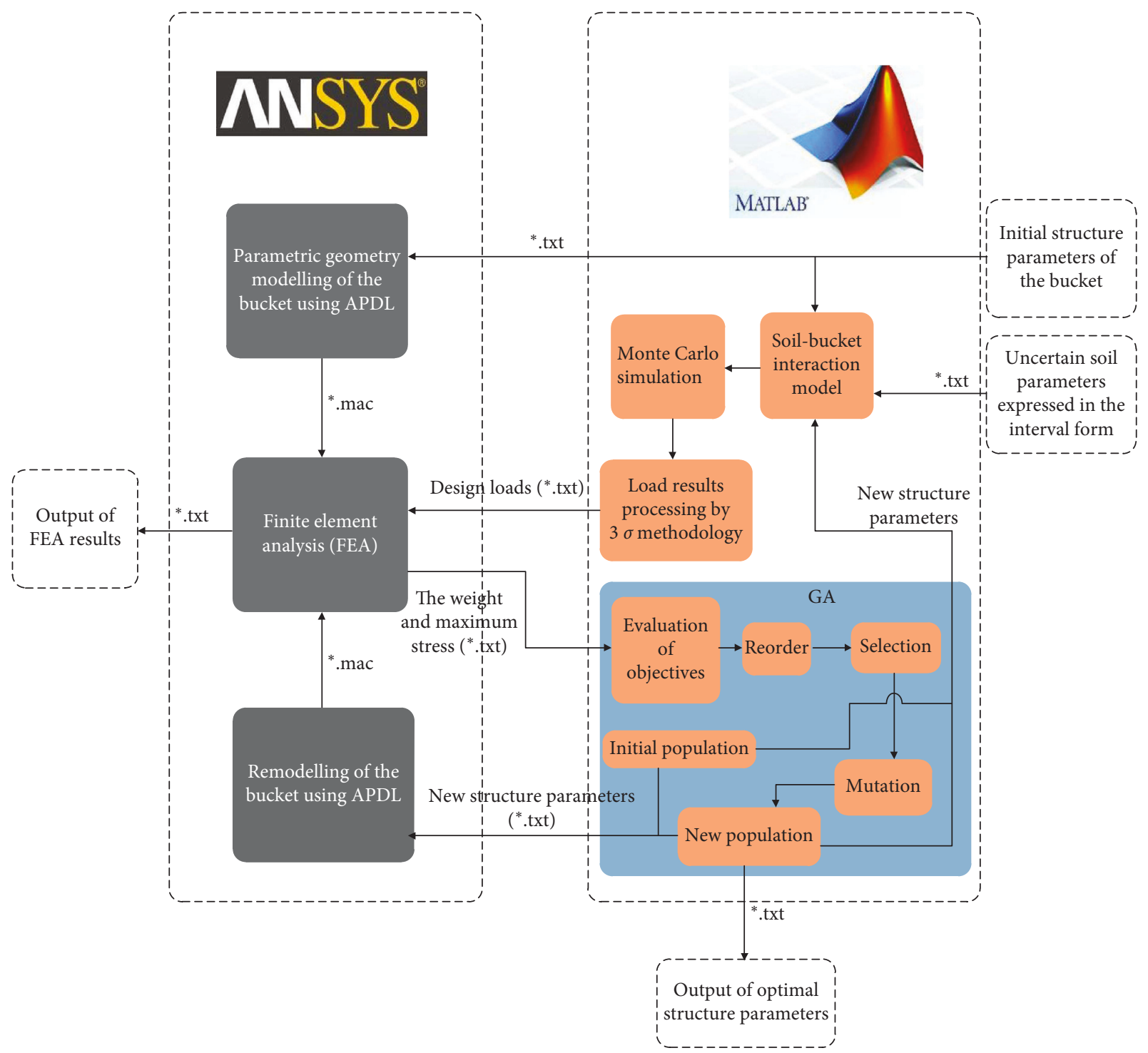

FIgURE 10: Framework of the bucket optimization. GA: genetic algorithm.

TABLE 5: Bounds of the continuous design variables based on the initial values.

\begin{tabular}{lc}
\hline Magnitude of design variables & Bounds \\
\hline $0 \sim 200$ & $\pm 20 \%$ of initial values \\
$>200$ & $\pm 10 \%$ of initial values \\
\hline
\end{tabular}

Table 6: Candidate values of the discrete variables.

\begin{tabular}{lc}
\hline Variable symbol & Candidate values $(\mathrm{mm})$ \\
\hline$t_{1}$ & {$[28: 1: 42]$} \\
$t_{2}$ & {$[13: 1: 19]$} \\
$t_{3}$ & {$[12: 1: 16]$} \\
$t_{4}$ & {$[20: 1: 30]$} \\
$t_{5}$ & {$[5: 1: 10]$} \\
\hline
\end{tabular}

the bucket volume capacity determined by all the design variables, and $V_{0}$ is its lower limit.

The set $\mathbf{X}$ includes continuous variables and discrete variables. Considering the accuracy of manufacture of steel, the thickness parameters $t_{i}(i=1,2, \ldots, 5)$ are set as discrete variables and are taken as integers, while the other variables are defined as continuous variables. Boundary conditions of the continuous variables are given in Table 5. Table 6 gives the candidate values of the discrete variables.

Mixed variable genetic algorithm is used to solve the optimization problem, since the discrete and continuous variables are included in the model. $S_{\text {max,ref }}$ and $m_{\text {ref }}$ are, respectively, taken as the initial maximum structure stress and the initial mass of the bucket. In the algorithm, the cross probability is 0.9 , the mutation is 0.05 , and the population size is 30 . The maximum number of generation is set as 50 . Each generation stands for a loop; thus, 50 loops are run in this optimization example. The initial and optimal values of design variables are listed in Table 7 . The optimal design variables are used to reconstruct the geometric model of the bucket and to reanalyse its strength. Table 8 gives the comparison of the sub-objectives.

It is obtained that the mass of the optimal bucket decreases by $11.11 \%$ relative to the initial bucket, and the 
TABLE 7: Initial and optimal values of design variables.

\begin{tabular}{|c|c|c|c|c|}
\hline Variable symbol & Initial value & Optimal value & Unit & Increment (\%) \\
\hline$\theta_{1}$ & 139 & 136.2 & $\circ$ & 2.01 \\
\hline$\theta_{2}$ & 24 & 22.5 & $\circ$ & -6.25 \\
\hline$\theta_{3}$ & 9 & 8.2 & $\circ$ & -8.89 \\
\hline$t_{1}$ & 35 & 39 & $\mathrm{~mm}$ & 11.43 \\
\hline$t_{2}$ & 16 & 17 & $\mathrm{~mm}$ & 5.88 \\
\hline$t_{3}$ & 14 & 14 & $\mathrm{~mm}$ & 0 \\
\hline$t_{4}$ & 25 & 20 & $\mathrm{~mm}$ & -20 \\
\hline$t_{5}$ & 8 & 5 & $\mathrm{~mm}$ & -37.5 \\
\hline$r_{1}$ & 372 & 350.5 & $\mathrm{~mm}$ & -5.78 \\
\hline$r_{2}$ & 114 & 96.9 & $\mathrm{~mm}$ & -15 \\
\hline$l_{1}$ & 270 & 292.7 & $\mathrm{~mm}$ & 8.41 \\
\hline$l_{2}$ & 546 & 501.2 & $\mathrm{~mm}$ & -8.21 \\
\hline$l_{3}$ & 580 & 542.7 & $\mathrm{~mm}$ & -6.43 \\
\hline$l_{4}$ & 601 & 645.8 & $\mathrm{~mm}$ & 7.45 \\
\hline$l_{5}$ & 458 & 461.9 & $\mathrm{~mm}$ & 0.85 \\
\hline$l_{6}$ & 68 & 60.7 & $\mathrm{~mm}$ & -10.74 \\
\hline$l_{7}$ & 110 & 112.3 & $\mathrm{~mm}$ & 2.1 \\
\hline$l_{8}$ & 446 & 480.6 & $\mathrm{~mm}$ & 7.76 \\
\hline$l_{9}$ & 495 & 489.5 & $\mathrm{~mm}$ & -1.11 \\
\hline$l_{10}$ & 263 & 249.1 & $\mathrm{~mm}$ & -5.29 \\
\hline$l_{11}$ & 175 & 180.7 & $\mathrm{~mm}$ & 3.26 \\
\hline$l_{12}$ & 1110 & 1004 & $\mathrm{~mm}$ & -9.55 \\
\hline
\end{tabular}

TABle 8: Comparison of the initial and optimal solutions.

\begin{tabular}{lccc}
\hline Sub-objective function & Initial value & Optimal value & Increment $(\%)$ \\
\hline Bucket weight $(\mathrm{kg})$ & 656.18 & 583.28 & -11.11 \\
Maximum stress $(\mathrm{MPa})$ & 387.43 & 263.89 & -31.89 \\
\hline
\end{tabular}

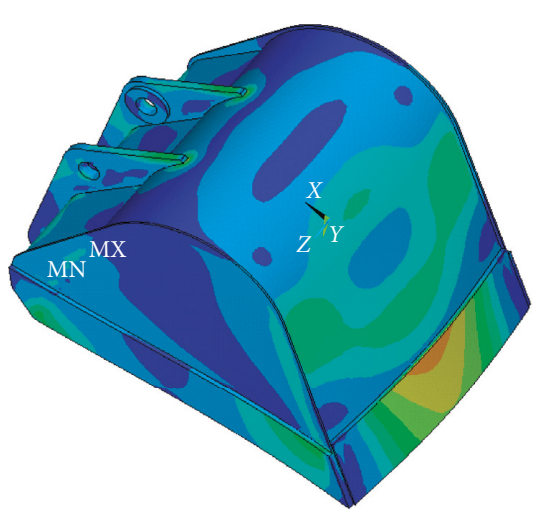

(a)

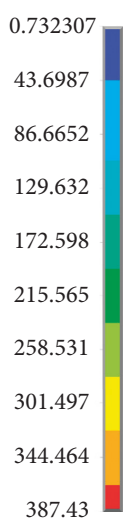

87.43

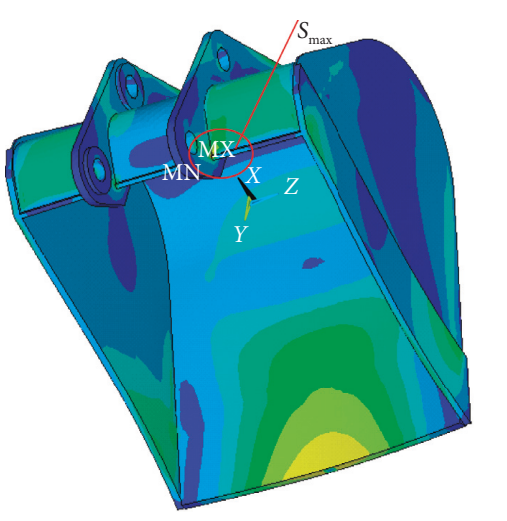

(b)

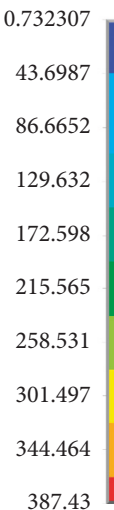

387.43

Figure 11: Initial FEA results based on design loads $(\mu+3 \sigma)$.

maximum stress also decreases by $31.89 \%$. This indicates that the proposed optimization methodology is effective to achieve the lightweight and high-strength design of the bucket. From Table 7, some variables have a large variation relative to their initial values but some other variables do not. All these optimal design variables can be used for remodelling the bucket structure based on APDL. In addition, in the optimization process the design loads are taken as the upper limits $\mu+3 \sigma$ of the forces acting on the bucket with the maximum digging depth and digging angle; thus, the safety probability of the optimal structure is 0.9987 . Figures 11 and 12, respectively, show the initial and optimal 

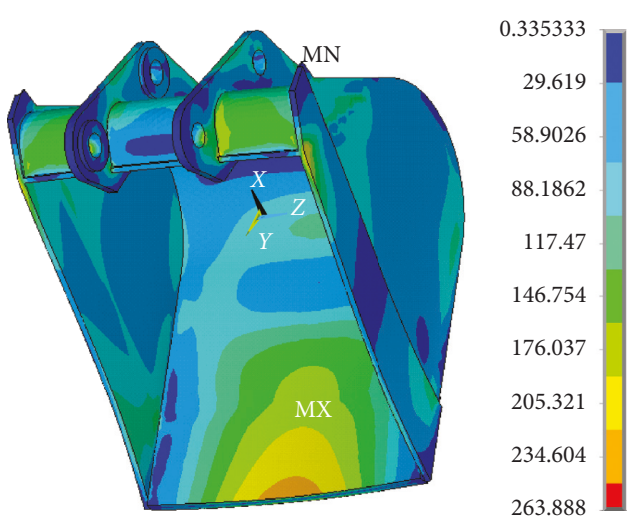

(a)

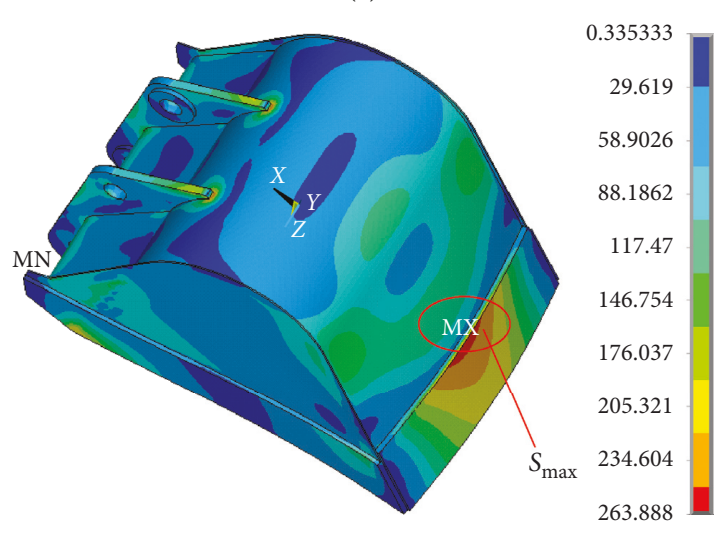

(b)

FIgURE 12: Optimal FEA results based on design loads $(\mu+3 \sigma)$.

results based on the design loads. The comparative results show that the optimized bucket decreases the structure stress remarkably. Thus, from the analysis results, it can be concluded that the proposed optimization method can achieve a good trade-off between the structure strength and the structure weight with the safety probability of 0.9987 .

\section{Conclusions}

In this paper, a framework to achieve high-strength and lightweight optimization design of the bucket under uncertain loading is introduced. A new design approach that can achieve good trade-off between the bucket weight and structure stress is also given. Since the novel method takes uncertainties inherent in the soil-bucket interaction model into account and uses 3-sigma methodology to quantify these uncertainties, it can ensure the optimized bucket has good working performance even if the working environment is variable and uncertain. The results of the supplied example also show that the optimized bucket has higher strength and lighter weight under uncertain loading. In the future work, the proposed framework will be used to develop a new bucket. Furthermore, the framework which can be used for the uncertain optimization design of the working attachment including the boom, stick, and bucket will be developed.

\section{Data Availability}

All the relevant data used to support the findings of this study are included within the article.

\section{Conflicts of Interest}

The authors declare that there are no conflicts of interest regarding the publication of this paper.

\section{Acknowledgments}

This study was supported by the National Natural Science Foundation of China (Grant no. 51475056). Grateful thanks go to Dr. Chen Jin for his writing guidance.

\section{References}

[1] ISO, Earth-Moving Machinery-Hydraulic Excavators and Manipulator Loaders-Methods of Determining Tool Forces, ISO, Geneva, Switzerland, 2006.

[2] Q. Qiu, B. Li, and P. Feng, "Optimal design of hydraulic excavator working device based on multiple surrogate models," Advances in Mechanical Engineering, vol. 8, no. 5, pp. 1-12, 2016.

[3] E. Rusinski, L. Cegiel, A. Michalczyk, P. Moczko, J. Olejarz, and D. Pietrusiak, "Investigation and modernization of buckets of surface mining machines," Engineering Structures, vol. 90, pp. 29-37, 2015.

[4] Z. Zou, J. Chen, and X. Pang, "Lightweight and high-strength optimization design for a fully parametric working attachment of a hydraulic excavator based on limiting theoretical digging capability model," Proceedings of the Institution of Mechanical Engineers, Part C: Journal of Mechanical Engineering Science, vol. 233, no. 14, pp. 4819-4835, 2019.

[5] A. Hadas, "Agricultural engineering soil mechanics: by E. McKyes. volume no. 10 in the series developments in agricultural engineering," Soil and Tillage Research, vol. 3-4, pp. 393-394, 1992.

[6] S. Blouin, A. Hemami, and M. Lipsett, "Review of resistive force models for earthmoving processes," Journal of Aerospace Engineering, vol. 14, no. 3, pp. 102-111, 2001.

[7] B. Park, Development of a virtual reality excavator simulator: a mathematical model of excavator digging and a calculation methodology, Ph.D thesis, Virginia Polytechnic Institute and State University, Blacksburg, VA, USA, 2002.

[8] A.-O. Kwame and F. Samuel, "Efficient cable shovel excavation in surface mines," Geotechnical and Geological Engineering, vol. 29, no. 1, pp. 19-26, 2011.

[9] C. P. Tan, Y. H. Zweiri, K. Althoefer, and L. D. Seneviratne, "Online soil-bucket interaction identification for autonomous excavation," in Proceedings of the 2005 IEEE International Conference on Robotics and Automation, pp. 3576-3581, ISIE, Barcelona, Spain, April 2005.

[10] S. Singh, "Learning to predict resistive forces during robotic excavation," in Proceedings of the 1995 IEEE International Conference on Robotics and Automation, pp. 2102-2107, ISIE, Nagoya, Aichi, Japan, May 1995.

[11] B. Nureddin, W. Ashwin, H. Meike et al., "Integration of digging forces in a multi-body-system model of an excavator," Proceedings of the Institution of Mechanical Engineers, Part K: Journal of Multi-body Dynamics, vol. 230, no. 2, pp. 159-177, 2016. 
[12] T. Ni, H. Zhang, C. Yu, D. Zhao, and S. Liu, "Design of highly realistic virtual environment for excavator simulator," Computers \& Electrical Engineering, vol. 39, no. 7, pp. 21122123, 2013.

[13] Y. B. Kim, J. Ha, H. Kang, P. Y. Kim, J. Park, and F. C. Park, "Dynamically optimal trajectories for earthmoving excavators," Automation in Construction, vol. 35, pp. 568-578, 2013.

[14] F. G. Flores, A. Kecskeméthy, and A. Pöttker, "Workspace analysis and maximal force calculation of a face-shovel excavator using kinematical transformers," in Proceedings of the 12th IFToMM World Congress in Mechanism And Machine Science, pp. 375-381, Besançon, France, June 2007.

[15] J. Chen, Z. Zou, and X. Pang, "Digging performance characterization for hydraulic excavator considering uncertainty during digging operation," Proceedings of the Institution of Mechanical Engineers, Part C: Journal of Mechanical Engineering Science, vol. 232, no. 5, pp. 857-871, 2018.

[16] S. Koji, O. Akira, and F. Kozo, "Development of multi-objective six-sigma approach for robust design optimization," Journal of Aerospace Computing, Information, and Communication, vol. 8, no. 5, pp. 215-233, 2008.

[17] S. Sundaresan, K. Ishii, and D. R. Houser, "A robust optimization procedure with variations on design variables and constraints," Engineering Optimization, vol. 24, no. 2, pp. 101-117, 2007.

[18] K. Ali, G. Liang, R. A. Farhan, and Q. Zeehan, “ \pm 3 -sigma based design optimization of 3D Finocyl grain," Aerospace Science and Technology, vol. 26, no. 1, pp. 29-37, 2013.

[19] T. Rashi, K. Jeremy, and D. George, "Bucket trajectory classification of mining excavators," Automation in Construction, vol. 31, pp. 128-139, 2013.

[20] K. Fujino, M. Moteki, A. Nishiyama et al., "Towards autonomous excavation by hydraulic excavator-measurement and consideration on bucket posture and body stress in digging works," in Proceedings of the 2013 IEEE Workshop on Advanced Robotics and its Social Impacts (Arso) Shibaura Institute of Technology, pp. 231-236, ISIE, Tokyo, Japan, November 2013.

[21] A. Stentz, J. Bares, S. Singh, and P. Rowe, "A robotic excavator for autonomous truck loading," Autonomous Robots, vol. 7, no. 2, pp. 175-186, 1999.

[22] J. V. Perumpral, R. D. Grisso, and C. S. Desai, "A soil-tool model based on limit equilibrium analysis," Transactions of the ASAE, vol. 26, no. 4, pp. 991-995, 1983. 


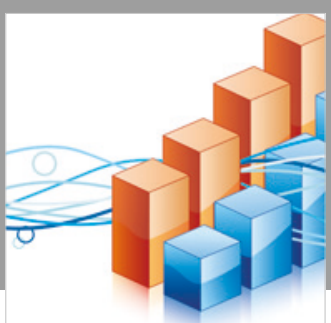

Advances in

Operations Research

\section{-n-m}
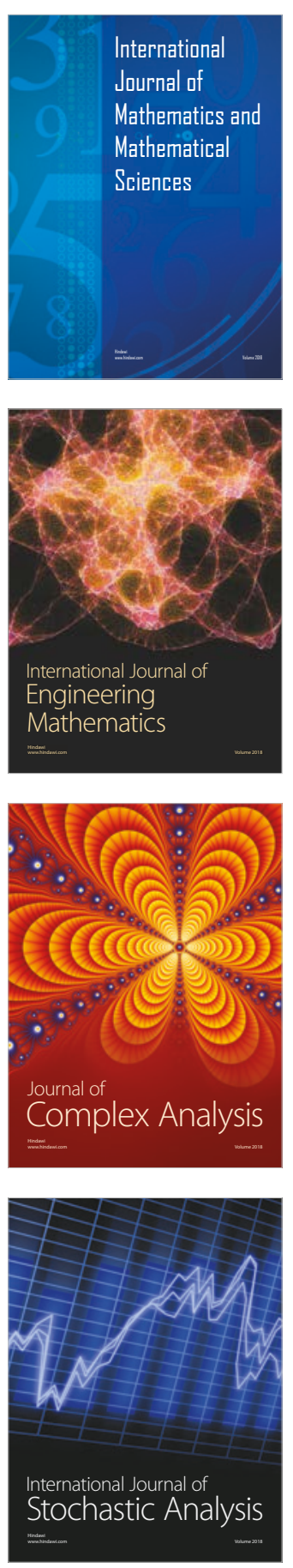
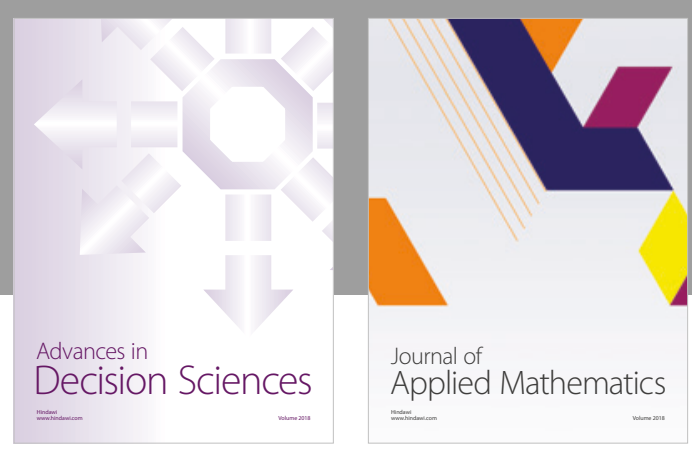

Journal of

Applied Mathematics
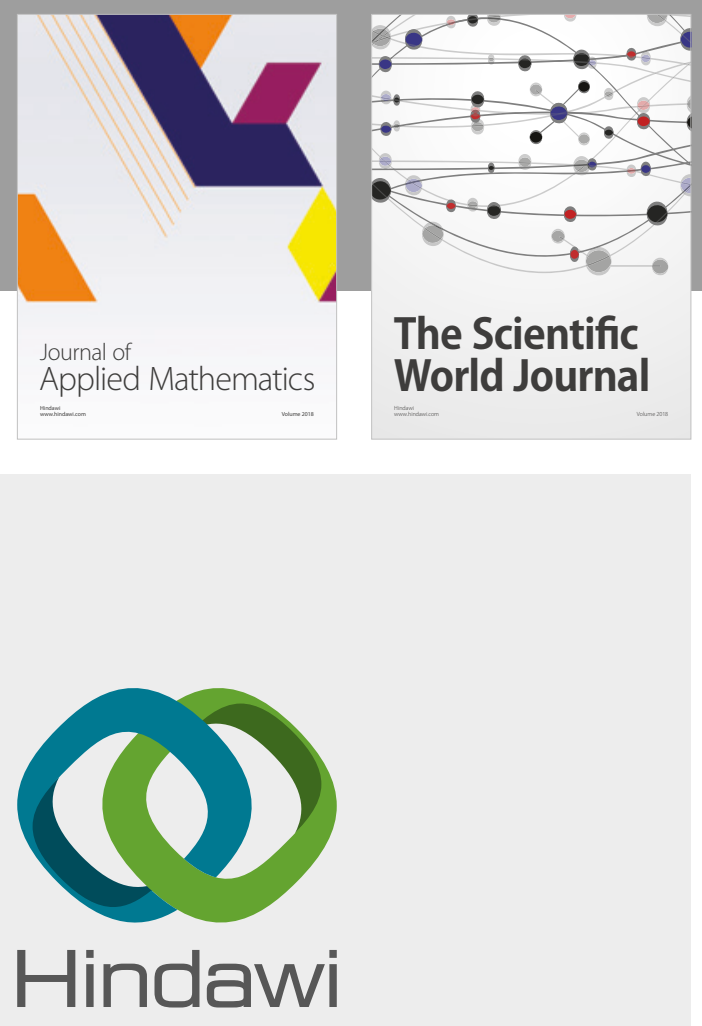

Submit your manuscripts at

www.hindawi.com

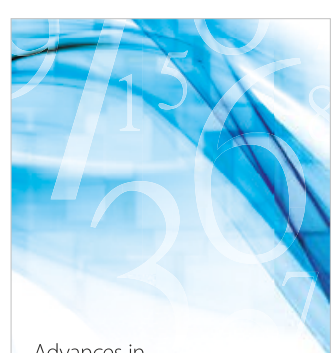

Advances in
Numerical Analysis
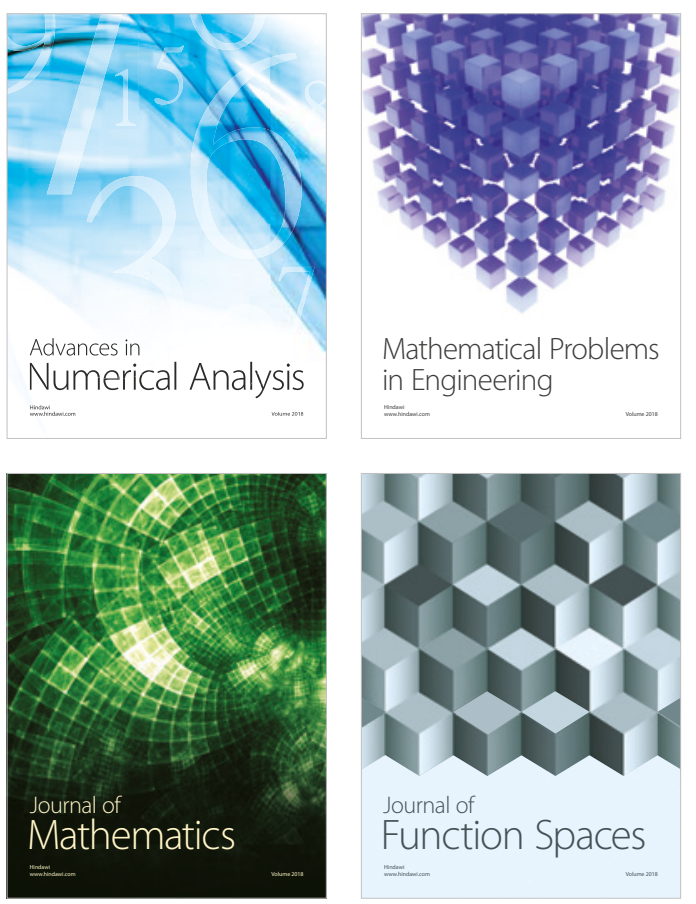

Mathematical Problems in Engineering

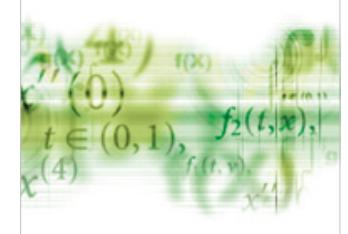

International Journal of

Differential Equations

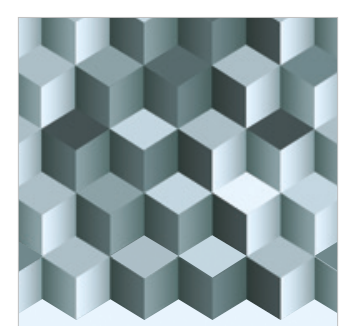

Journal of

Function Spaces

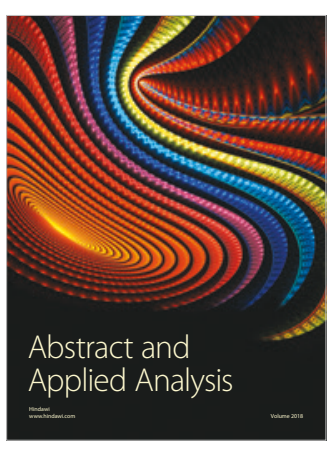

The Scientific

World Journal

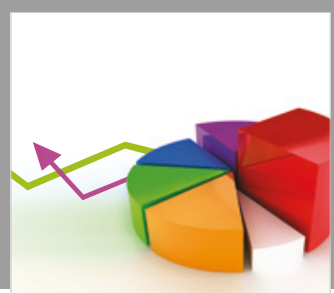

Journal of

Probability and Statistics
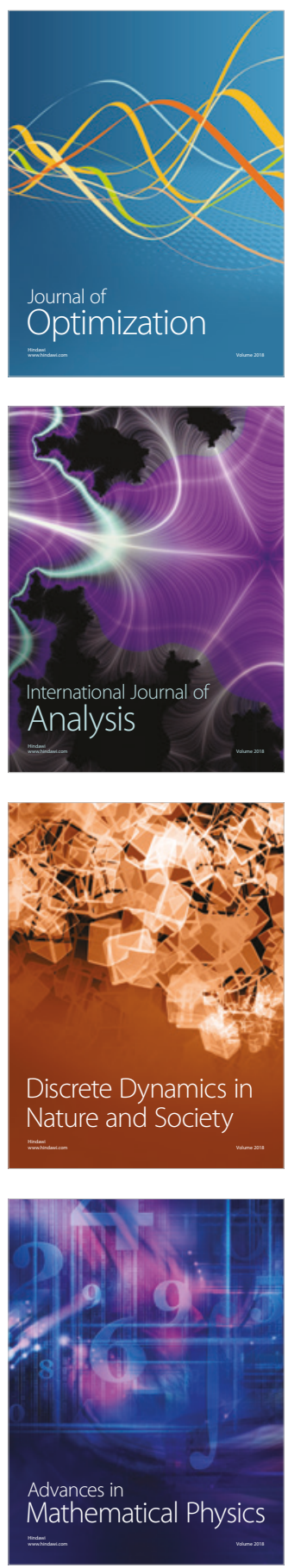